\title{
PROGRESS
}

\section{Structural vaccinology starts to deliver}

\author{
Philip R. Dormitzer, Guido Grandi and Rino Rappuoli
}

\begin{abstract}
Following the impact of the genomics revolution on vaccine research and the development of reverse vaccinology, it was predicted that another new approach, structure-based antigen design, would become a driving force for vaccine innovation. Now, 5 years on, there are several examples of how structure-based design, or structural vaccinology, can deliver new vaccine antigens that were not possible before. Here, we discuss some of these examples and the contribution of structural vaccinology to our understanding of the protective epitopes of important bacterial and viral pathogens.
\end{abstract}

Vaccines have been instrumental in conquering many infectious diseases, and they have contributed greatly to the increased life expectancy and quality of life that we now take for granted in modern societies. In the twenty-first century, new vaccine technologies have the potential to make continued important contributions to improving our quality of life by conquering those diseases that could not be defeated with older technologies ${ }^{1}$. The list of new vaccines needed by modern societies includes vaccines against those pathogens that cause the remaining infectious diseases of infants and neonates, such as the serogroup B meningococcus (MenB; Neisseria meningitidis serogroup B), respiratory syncytial virus (RSV), group A Streptococcus (GAS; Streptococcus pyogenes) and group B Streptococcus (GBS; Streptococcus agalactiae). It is also becoming increasingly important to develop vaccines against antibiotic-resistant bacteria such as Staphylococcus aureus, Clostridium difficile and Pseudomonas aeruginosa. In addition, we still need vaccines for some of the most difficult infectious diseases to target, such as tuberculosis, malaria, HIV/AIDS and hepatitis $\mathrm{C}$ virus (HCV) infection, and we also need vaccines against emerging diseases, such as pandemic influenza ${ }^{1}$.

In the late 1990s, the development of high-throughput DNA sequencing allowed entire bacterial genomes to be sequenced, providing access to the complete antigenic repertoire of bacterial pathogens and facilitating the discovery of many previously unknown vaccine antigens through a process known as reverse vaccinology ${ }^{2}$. Over the past decade, advances in X-ray crystallography and NMR spectroscopy have increased the throughput of protein structure determination so that today we can determine the structure of most vaccine antigens and their epitopes. Five years ago, we predicted that this knowledge would form the basis for another major change in vaccine research and development, with the beginning of the era of structural vaccinology, in which three-dimensional (3D) structural information could be used to design novel and improved vaccine antigens $^{3}$. In this Progress article, we discuss the first published reports of structure-based antigen design, demonstrating that structural vaccinology is becoming a reality. In the near future, it may be possible to use this technique to design fully synthetic and universal vaccines and also to obtain a fuller understanding of the nature of the antigenic epitopes that are recognized by the human immune system.

\section{MenB fHbp}

Licensed vaccines against $N$. meningitidis serogroups A, C, Y and W135 are composed of capsular polysaccharides usually conjugated to a carrier protein. However, carbohydrate and glycoconjugate vaccines cannot be used to target MenB because the capsular polysaccharide of this serogroup is chemically identical to a human self-antigen. Recently, potential antigens that could be used in a vaccine against MenB have been discovered based on the determination of the MenB genome sequence. One of these protein antigens is a surface-exposed lipoprotein, factor $\mathrm{H}$-binding protein $(\mathrm{fHbp})^{4-7}$. This antigen is very effective at eliciting protective antibodies, but it has more than 500 known amino acid sequence variants (see http://pubmlst.org/neisseria/fHbp). These variants can be classified into two ${ }^{6}$ or three ${ }^{4}$ distinct variant groups (hereafter referred to as variants 1,2 and 3) that do not induce cross-protective immunity to each other, as measured by an in vitro bactericidal assay in which antibodies mediate complementdependent bacterial killing. To induce strong protective immunity against all three variants, antigens specific for each variant must be included in the vaccine, which makes the manufacturing process complex and expensive. Therefore, the $N$. meningitidis vaccines that are currently in clinical trials include either two fHbp variants ${ }^{6}$, or only the fHBP variant from the most abundant group (variant 1 ) in combination with other antigens to cover strains that express the other variants ${ }^{8}$.

A single antigen that could induce immunity against all the fHbp sequence variants would be an ideal vaccine candidate. Such an antigen has been created through structurebased design: epitopes from each of the three fHbp antigenic-variant groups were engineered into a single molecule. The 3D structure of fHbp was determined by NMR and $\mathrm{X}$-ray crystallography, revealing a core structure consisting of two $\beta$-barrels connected by a short linker ${ }^{9-11}$. The epitopes recognized by protective monoclonal antibodies against each of the three antigenic variants were then determined ${ }^{12,13}$. The protective epitopes of variant 1 and of variants 2 and 3 map in nonoverlapping regions located mostly in the amino- and carboxy-terminal regions of $\mathrm{fHbp}$, respectively. Interestingly, the epitopes of variant 1 and variants 2 and 3 overlap in the primary protein sequence but not in the $3 \mathrm{D}$ structure. The first approach used to create a single cross-protective antigen was to engineer the variant 1 gene to contain codons for the amino acids that are essential for the recognition of variants 2 and 3 . This approach failed, and it became clear that to 


\section{PROGRESS}

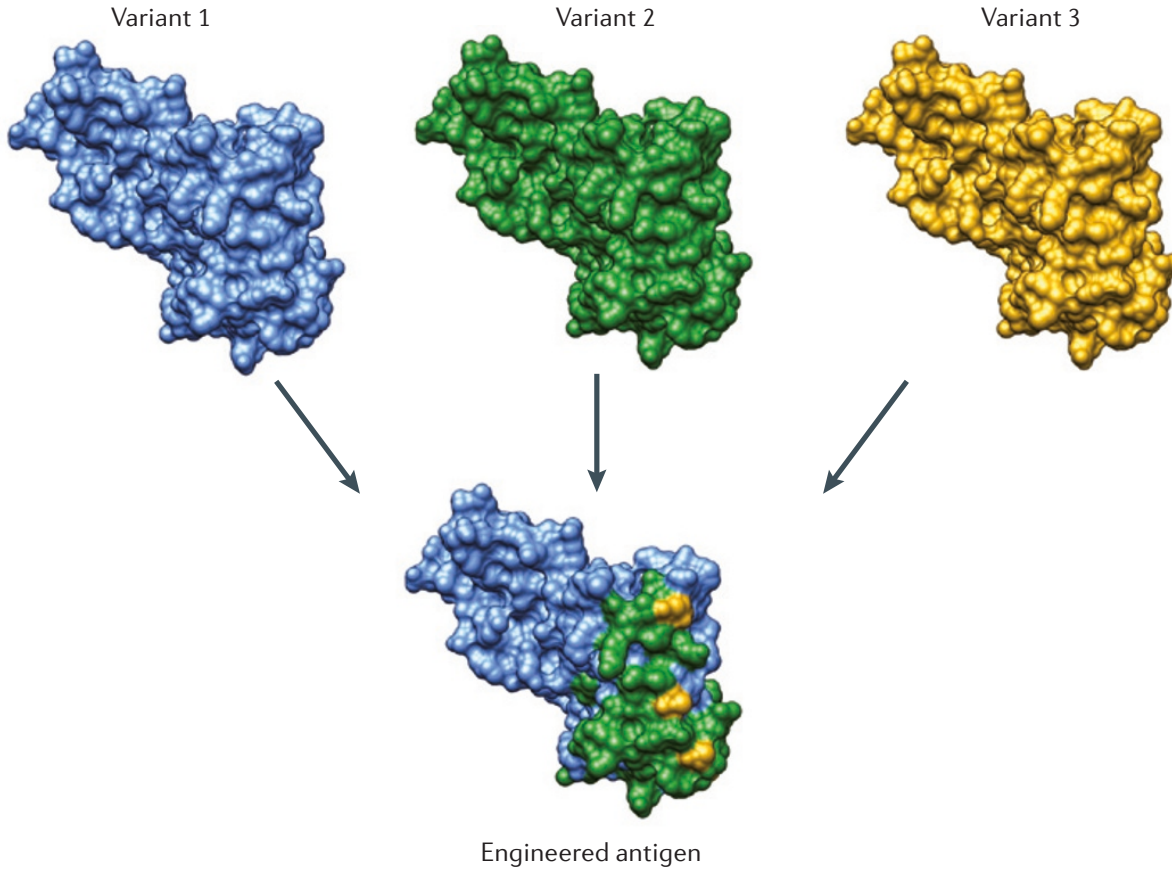

Figure 1 | Rational design of a cross-protective factor $\mathrm{H}$-binding protein. The structure of factor $\mathrm{H}$-binding protein variants 1,2 and 3 from the serogroup $B$ meningococcus (Neisseria meningitidis serogroup B), and the engineered antigen for the vaccine. A cluster of amino acids from the surface of variants 2 and 3 was engineered into the variant 1 structure to generate a chimeric molecule that elicits immunity against all three variants. In the antigen, the patch of the protein surface that matches variant 2 is coloured green (this patch includes both residues that are specific to variant 2 and those that are shared with variants 1 and 3 ), whereas residues that are specific to variant 3 are coloured yellow, and residues that are specific for variant 1 are coloured blue.

elicit protective antibodies it would be necessary not only to introduce the key amino acids from each epitope but also to recreate the entire epitope surface, strictly mimicking its $3 \mathrm{D}$ structure in the native molecule, and a large-scale design effort was started ${ }^{5}$. The C-terminal region of variant 1 was divided into ten areas large enough to contain at least one conformational epitope (approximately 900-2,000 $($ REF. 2)), and the residues of the variant $1 \mathrm{C}$ terminus were then replaced with the corresponding amino acids of variants 2 and/or 3, regardless of their position in the primary sequence (FIG. 1). To preserve folding, amino acid substitutions were introduced only for residues with side chains that are well exposed to solvent, leaving the internal core of the protein unaltered. Fifty-four molecules were designed, approximately five molecules for each of the ten areas identified. The molecules were then expressed, purified and used to immunize mice, and the sera generated were tested for the presence of bactericidal antibodies against MenB strains carrying fHbp variants 1,2 and 3 . Several molecules elicited a broad immune response. One was selected for further study and was shown to elicit bactericidal antibodies against MenB strains carrying fHbp variants 1, 2 and 3 (REF. 5). The crystal structure of the successful molecule showed complete conservation of the native fold, making it an ideal candidate for the next generation of meningococcal vaccines.

The main lessons learned from this work are that the epitopes recognized by antibodies are larger than the 'patches' of amino acids that can be identified using conventional epitope mapping, and that the individual amino acids identified as being essential for an epitope can be less important than preserving the overall epitope surface $^{5,13}$. $\mathrm{fHbp}$ is the target of several other projects using structure-based design to improve the immunogenicity of the protein or the breadth of the immune response that it stimulates ${ }^{11,14,15}$. For example, it has been proposed that removing factor $\mathrm{H}$-binding activity could improve fHBP immunogenicity in humans, so several fHbp mutants that are unable to bind factor $\mathrm{H}$ have been generated, and one of these has been shown to be more immunogenic than wild-type $\mathrm{fHbp}$ in rats that are transgenic for human factor $\mathrm{H}^{14}$. In another case, the breadth of the immune response generated has been improved by fusing the $\mathrm{N}$ terminus of $\mathrm{fHbp}$ variant 1 to the $\mathrm{C}$ terminus of $\mathrm{fHbp}$ variant 2 (REF. 16).

\section{GBS pilus BP}

GBS causes serious infections in 30-300 newborns for every 100,000 births ${ }^{17}$. In most industrialized countries, pregnant women colonized with GBS are treated with antibiotics during childbirth, and this practice has reduced but not eliminated the number of neonate infections. Because babies delivered by women with high titres of GBS-specific opsonophagocytic antibodies are protected from GBS infection ${ }^{18}$, vaccination of women of childbearing age and/or pregnant women appears to be an ideal longlasting solution to the problem of neonate infection. However, for several reasons, an effective anti-GBS vaccine is not yet available. One reason is the fact that there are ten GBS serotypes, necessitating a complex vaccine if serotype-specific immunogens are selected.

It was recently shown that all GBS strains express pili, which are long filamentous structures involved in bacterium-host interactions, bacterial aggregation and biofilm formation ${ }^{19,20}$. GBS pili are composed of three protein subunits: backbone protein (BP), which forms the pilus shaft; ancillary protein 1 (AP1), which decorates the pilus stem; and AP2, which is often found at the base of the pilus and anchors it to the cell wall. All three proteins are covalently linked to each other through a sortase-mediated transpeptidation reaction ${ }^{21}$. BP proteins induce protective immunity, suggesting that they can be used as vaccine antigens. However, there are three genomic pilus islands (PI-1, PI-2a and PI-2b) that each encode a complete set of pilus proteins, and each GBS strain can carry one or two islands ${ }^{22}$. Furthermore, the BP encoded by PI-2a (BP-2a) has six sequence variants ${ }^{22}$, suggesting that antigens from eight BPs would be required to make a vaccine that was effective against all GBS strains. This is a challenge. The $3 \mathrm{D}$ structure ${ }^{23}$ of one of the six BP-2a variants suggested a possible solution, as it revealed a four-domain organization in which domain three (D3), which is 100 amino acids long, is likely to face the external side of the pilus shaft, based on the capacity of D3-specific antibodies to bind GBS. D3 elicits high titres of opsonophagocytic antibodies, which protect mice against lethal challenge with GBS isolates expressing the PI-2a pilus. The 3D structure was used to model the structures of the other five BP-2a variants and confirmed that all BP-2a variants have 


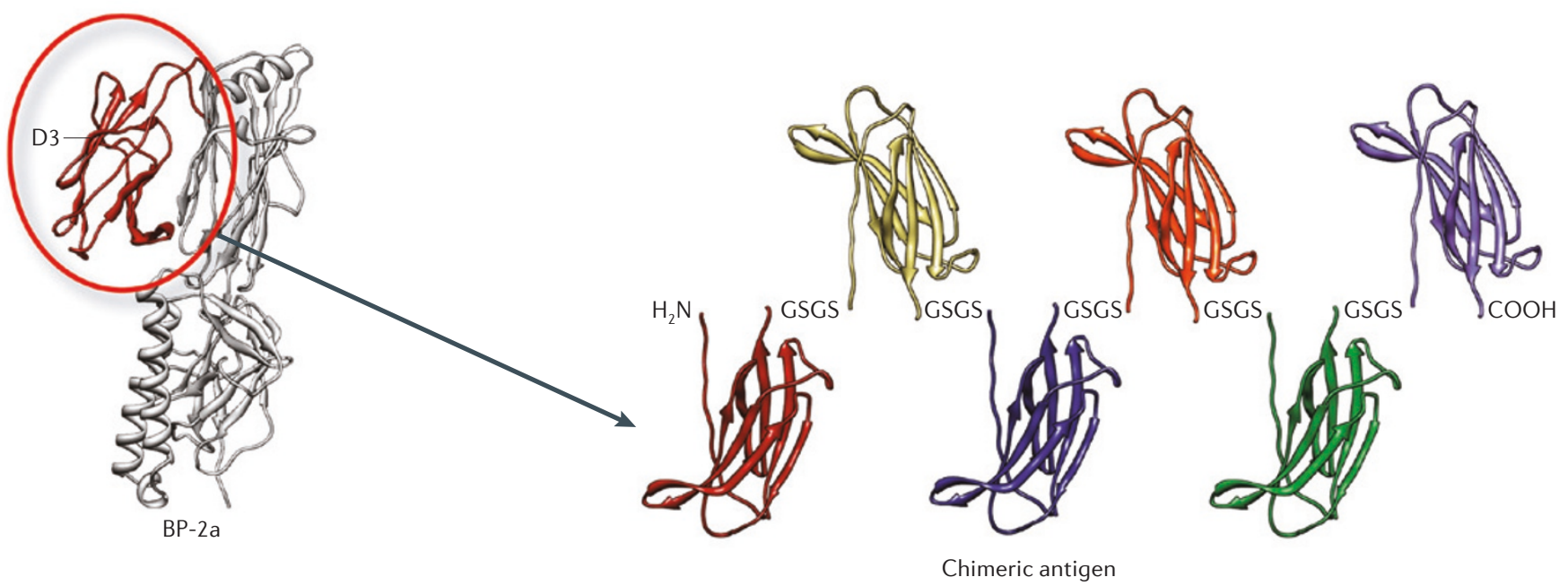

Figure 2 | Targeting the group B Streptococcus pilus backbone protein. A chimeric antigen based on domain three (D3) of the group B Streptococcus (GBS; Streptococcus agalactiae) type 2a pilus backbone protein (BP-2a) variants is shown. The concatamer of D3 antigenic variants, connected by a Gly-Ser linker (GSGS) was designed to elicit broad immunity against GBS strains. a similar four-domain structural organization. Immunization with recombinant D3 domains from the other BP-2a variants also proved to be highly protective against challenge with GBS cells expressing the respective pili. These experiments led to the important observation that the protective capacity of a BP-2a variant is restricted to the D3 region of that variant (FIG. 2). Because of their small size, the D3 domains from each variant could be fused into a single recombinant construct that is efficiently expressed in Escherichia coli and can be purified. The recombinant chimaera (FIG. 2) confers strong protection against all strains expressing a BP-2a variant ${ }^{23}$. This structure-based work might pave the way for the development of a universal, broadly protective GBS vaccine. In more general terms, this work revealed that specific structural domains within a protein can sometimes be sufficient to elicit a protective immune response.

\section{RSV F glycoprotein}

RSV infects the lower respiratory tract of most infants and children and is often associated with hospitalization ${ }^{24}$. The virus is a difficult vaccine target. An early formalininactivated RSV vaccine candidate actually enhanced subsequent RSV-mediated disease ${ }^{25}$; live attenuated vaccines have yet to strike an acceptable balance between over- and under-attenuation ${ }^{26}$; and subunit vaccines have proved to be biochemically challenging to develop.

The obvious target for RSV subunit vaccines is the fusion (F) glycoprotein ${ }^{27,28}$ (FIG. 3a). This trimeric protein forms a lollipop-shaped structure on the virion (FIG. 3b), with a C-terminal triple coiled-coil domain anchored in the virion membrane ${ }^{27-30}$. Palivizumab, a human monoclonal antibody against this protein, neutralizes the virus and is licensed for prophylaxis against RSV infection in high-risk infants. During RSV entry into cells, the RSV F protein, like other paramyxovirus $\mathrm{F}$ proteins, rearranges from the pre-fusion form through an intermediate extended structure to a post-fusion state ${ }^{27-31}$ (FIG. 3b,c). During this rearrangement, the C-terminal coiled-coil dissociates into its three constituent strands, which then wrap around the globular head and join three additional helices to form the post-fusion six-helix bundle. Thus, the six-helix bundle 'stem' of the crutch-shaped post-fusion molecule (FIG. 3c) is on the opposite side of the globular head from the three-helix coiledcoil stem of the pre-fusion molecule (FIG. 3b). As a result of these molecular gymnastics, the viral membrane (traversed by the $\mathrm{F}$ transmembrane domain) and the host cell membrane (into which the F fusion peptide has inserted) are brought into close approximation, facilitating membrane fusion and virus entry.

On initial inspection, the desired conformer of $\mathrm{F}$ for use as a vaccine antigen seems obvious. The pre-fusion form is displayed on infectious virions, and from its gross contours looks very different from the post-fusion form ${ }^{30}$ (FIG. 3b,c). However, producing a subunit vaccine antigen corresponding to the pre-fusion conformer has proved difficult. The pre-fusion form flips to the post-fusion state when it is extracted from a membrane with detergent or when expressed as a non-membrane-anchored ectodomain ${ }^{32}$. The extended intermediate form is transient, inferred rather than observed and an unlikely candidate for a vaccine antigen. The post-fusion form has difficult biochemical characteristics, as it is, essentially, a detergent, with one hydrophobic end and one hydrophilic end. With difficulty, native post-fusion F can be semipurified. However, electron microscopy of post-fusion F prepared by gentle means revealed amorphous globules decorated with crutch-shaped F spikes, presumably corresponding to lipid droplets solubilized by an F 'detergent' (P.R.D., unpublished observations). Given the conformational heterogeneity of $\mathrm{F}$ and its detergent-like properties, it is not surprising that preparations extracted from RSV virions have proved difficult to use as reproducible, homogeneous vaccine antigens ${ }^{33,34}$.

Structural insights from studies on the related human parainfluenza virus 3 (HPIV-3) and parainfluenza virus 5 (PIV-5) $\mathrm{F}$ glycoproteins have guided engineering of RSV $F^{29,31}$. Elimination of the transmembrane and cytoplasmic regions to produce an ectodomain was an obvious first step (FIG. 3a). Subsequent manipulation of the fusion peptide region to reduce hydrophobicity was less obvious. A molecule in which the fusion peptide had been deleted might have misfolded owing to the pre-fusion protein lacking the interactions of the fusion peptide within the globular head. However, RSV F has substantial differences from the parainfluenza virus $\mathrm{F}$ proteins in this region, and partial deletion of the fusion peptide from the RSV F ectodomain resulted in the formation of a hydrophilic, very stable, homogeneous post-fusion trimer ${ }^{27}$. This 


\section{PROGRESS}

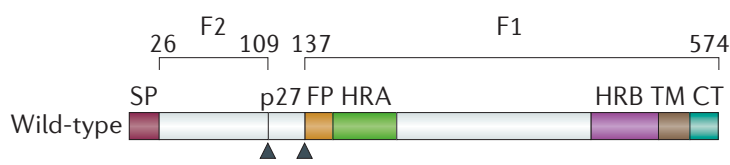

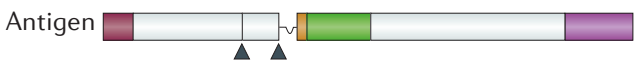

b Pre-fusion $\mathrm{F}$

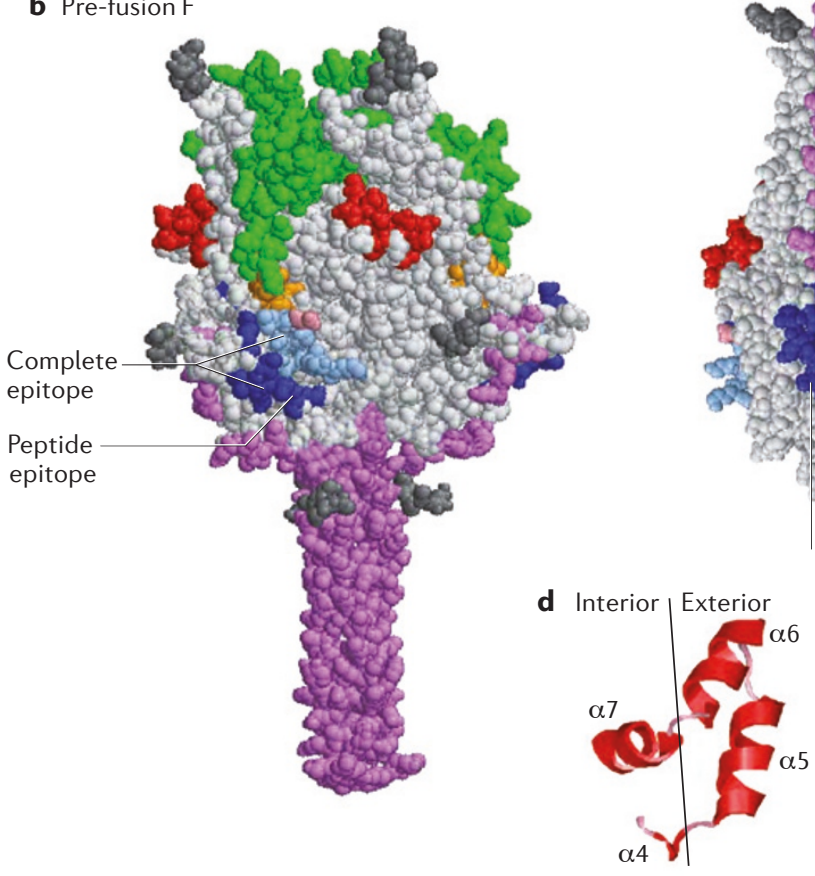

RSV
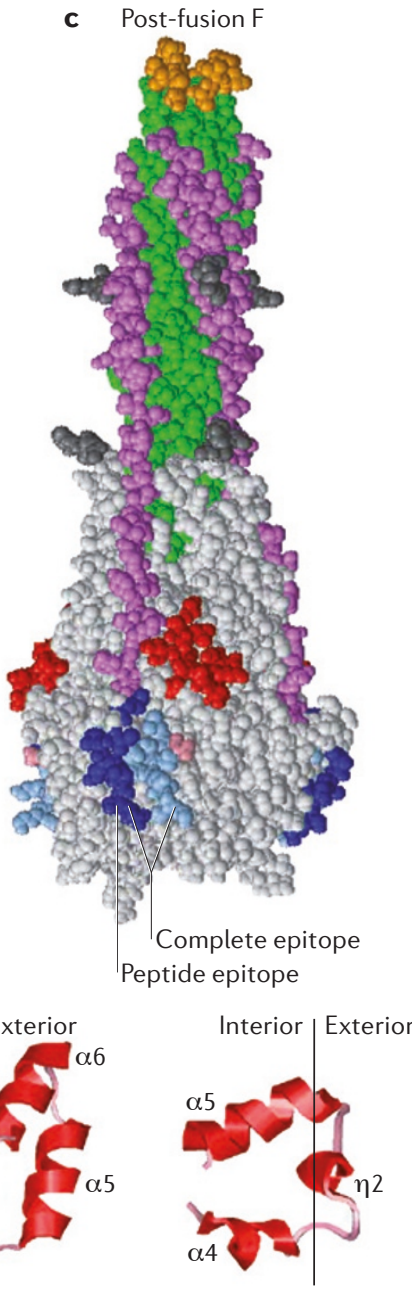

HPIV-3

Figure 3 | Targeting the respiratory syncytial virus fusion protein. a |A comparison of wild-type fusion (F) protein from respiratory syncytial virus (RSV) with the engineered RSV F antigen. Arrowheads indicate furin cleavage sites, and the peptide p27 is released after cleavage. The F1 and F2 fragments of the wild-type sequence, which are produced as a result of furin cleavage, are indicated. b $\mid$ A model of the engineered RSV pre-fusion F, based on data from parainfluenza virus 5 (PIV-5) prefusion $\mathrm{F}$ and engineered RSV post-fusion $\mathrm{F}$ crystal structures. The carboxy-terminal coiled-coil region, which includes heptad repeat $B(H R B)$, is shown in violet. Globular-head residues (HRA in part a) that will extend to form the coiled-coil at the centre of the post-fusion six-helix bundle are shown in green. Residues from epitope A, the motavizumab and palivizumab epitope, that were included in the peptide approximation of the epitope are shown in red; the extended epitope $A$ also includes the isolated pink residue. Residues from epitope $\mathrm{C}$, the $101 \mathrm{~F}$ epitope, that were included in the peptide approximation of the epitope are shown in dark blue; the extended epitope also includes the residues in light blue (the pink residue is also in the extended epitope C). The remnant of the fusion peptide (FP) is shown in orange, and glycans are shown in dark grey. $\mathbf{c}$ |The engineered RSV post-fusion F structure. d) The core post-fusion structure of the motavizumab and palivizumab epitope of RSV F (based on Protein Data Bank (PDB) accession 3RKI), and the homologous post-fusion structure of $\mathrm{F}$ from human parainfluenza virus 3 (HPIV-3) (based on PDB accession 1ZTM). The RSV structure has a short helical insertion, $\alpha 7$, that is not present in HPIV-3 F and that forces the helix-turn-helix ( $\alpha 5$ and $\alpha 6$ ) epitope to the surface in RSV F. The structural models were generated using Rasmol. CT, cytoplasmic tail; SP, signal peptide; TM, transmembrane region.

engineered RSV F is hydrophilic enough to be readily purified and formulated as a vaccine antigen, stable enough to heat to above $90^{\circ} \mathrm{C}$ without apparent disruption, and homogeneous enough to crystallize.
From a biochemical perspective, the engineered post-fusion $\mathrm{F}$ is a highly desirable vaccine antigen with the potential for efficient and consistent production, easy characterization and room temperature stability. But the post-fusion conformation of full-length F appears to be considerably different from the pre-fusion form, which is thought to be the dominant conformer on the virion. If neutralizing and protective antibodies function by interfering with virus entry, as is thought to be the case, then antibody recognition of a conformer that forms only after membrane fusion would not be useful in protection. Indeed, structural modelling based on the known PIV-5 pre-fusion F structure predicts that the key epitope recognized by the potent neutralizing antibodies palivizumab and motavizumab is buried in the interface between the pre-fusion RSV F trimer subunits and not accessible for antibody binding ${ }^{35}$, and most of the corresponding residues of HPIV-3 $\mathrm{F}$ are buried in the post-fusion crystal structure $^{31}$ (FIG. 3d). However, immunization of animals with the engineered RSV post-fusion F elicits high titres of RSV-neutralizing antibodies, some of which compete for binding with motavizumab ${ }^{27}$.

The crystal structure of the engineered RSV post-fusion $\mathrm{F}$ antigen explains one aspect of the unanticipated efficiency of this antigen in eliciting neutralizing antibodies $^{27,28}$. On first inspection, the engineered RSV post-fusion F structure appears to be almost identical to that of HPIV-3 post-fusion protein. However, there is a subtle difference: the engineered RSV F has a six-residue $\alpha$-helix ( $\alpha 7)$ inserted adjacent to the helix-turn-helix ( $\alpha 5$ and $\alpha 6$ ) motif recognized by motavizumab (FIG. $3 d$ ). This insertion, which was not predicted by homology modelling, forces residues from the motavizumab (and palivizumab) epitope out from their buried position in the intersubunit interface and onto the solventexposed surface, where they are accessible to antibody. A combination of the determined structure for the engineered RSV post-fusion F protein and modelling based on the PIV-5 pre-fusion F structure revealed that, although $\mathrm{F}$ proteins undergo a dramatic gross rearrangement from pre-fusion to post-fusion, this rearrangement consists of refolding of a limited number of structural elements. Thus, the best characterized neutralizing epitopes (those that have been determined from known antibody-peptide structures and from single point mutations that result in escape from monoclonal antibody neutralization) are well preserved between the pre- and post-fusion forms. The engineered post-fusion form of the $\mathrm{F}$ protein is now approaching clinical trials in a vaccine against RSV. 
Lessons from RSV. Observations made regarding the RSV F protein epitopes that are recognized by motavizumab and $101 \mathrm{~F}$ (another monoclonal antibody) correlate well with the observations made for MenB $\mathrm{fHbp}$, for which the extended surface of an intact, folded domain had to be engineered to obtain an immunogen that elicited functional antibodies. Motavizumab recognizes peptide 254-277 (red in FIG. 3b,c), and 101F recognizes peptide $422-438$ (dark blue in FIG. 3b,c) of RSV F ${ }^{35,36}$. The conformations of synthetic peptides (corresponding to these two regions) bound to the antigen-binding fragments of the antibodies, as determined by X-ray crystallography, are similar to the conformations of the same residues in the native protein, adopting a helix-turn-helix (FIG. 3d) and an extended coil (not shown), respectively ${ }^{27,28}$. However, despite having similar conformations in the co-crystal structures, the two isolated synthetic peptides bind the antibodies with affinities that are 6,000-fold (for motavizumab) and 16,000 -fold (for 101F) lower than the affinity of full-length F glycoprotein ${ }^{35,36}$. One explanation for this finding is that there are additional residues in $\mathrm{F}$ that are not included in the synthetic peptides but bind the antibodies (FIG. 3b,c). In the case of motavizumab, structural and functional evidence indicates that, at least in pre-fusion $\mathrm{F}$, the antibody contacts not only the residues of peptide254-277 but also residue 454, located in an F subunit adjacent to the one containing the helix-loop-helix motif. Therefore, the optimal antibody-binding surface has a quaternary conformation involving two subunits of the trimer ${ }^{28}$. Contact with an additional residue, amino acid 465, is also possible, but experimental confirmation is lacking. Similarly, in the case of $101 \mathrm{~F}$, the antibody contacts not only the residues peptide $422-438$ but also amino acids 418-421 and 451-456 of the same subunit ${ }^{28}$ (light blue in FIG. $3 d$ ). An alternative explanation is that the overall $\mathrm{F}$ protein fold is necessary to scaffold the peptides, locking them in the correct conformation to allow high-affinity interactions with antibodies. It is likely that the isolated peptides are floppy and adopt many conformations, but that the bound antibody locks them in the conformation observed in the co-crystal structures $^{37,38}$.

Consistent with their low affinity for motavizumab, when synthetic peptides corresponding to the contiguous sequence at the core of the motavizumab epitope are used as immunogens, they fail to elicit neutralizing antibodies ${ }^{37}$. Indeed, immunization of rabbits with such peptides elicits antisera that recognize the peptides but fail to recognize RSV itself. When floppy peptides are used to immunize animals, these peptides can gyrate through many conformational states, binding many B cell receptors and not just those that recognize the peptide conformer matching the state of the corresponding residues in the native protein. Thus, clonal expansion and affinity maturation is not restricted to $\mathrm{B}$ cells bearing virus-neutralizing antibodies, and there is no significant increase in the proportion of virus-neutralizing antibodies in the sera of immunized animals. When a peptide at the core of the motavizumab epitope was engineered into protein scaffolds that were designed to maintain the peptide in the native conformation, the affinity of the peptide for the antibody increased only threefold, and the immunogen still failed to elicit neutralizing antibodies, reflecting the difficulty of engineering truly authentic mimics of intact domain and epitope structure ${ }^{39}$.

These examples confirm that the peptides that we usually call epitopes, because they are recognized in vitro by antibodies, are imperfect mimics of the real surfaces recognized by antibodies in immunized or infected hosts. The peptides probably contain key residues that form the epitope, but they might lack additional residues that are outside of the linear epitope; they usually lack a single, defined conformation; they might bind functional antibodies with affinities that are several orders of magnitude lower than the affinity of the native protein; and they might preferentially elicit antibodies that recognize different structural aspects than those recognized by protective antibodies. Therefore, the future of structural vaccinology is in the engineering of antigenic surfaces on domains rather than the use of isolated epitopes.

\section{Influenza virus HA}

The examples that we cite above demonstrate the use of structural vaccinology to improve the biochemical characteristics of vaccine antigens and to guide the combination of epitopes that is necessary to increase the breadth of the immune response. In the future, a better understanding of how protein structures interact with the cells of the immune system during antigen uptake, processing and presentation, during clonal expansion of B cells and during antibody affinity maturation could allow a more sophisticated use of structural biology to guide antigen design ${ }^{3}$. For instance, recent work shows that the germline-encoded antibody precursor against an HA epitope has such a low affinity for this epitope that it can only bind the antigen in the membranebound pentameric immunoglobulin $\mathrm{M}$ conformation, and that only 7 amino acid changes were necessary to obtain a high-affinity antibody ${ }^{40}$. In complex multiepitope antigens, such as influenza virus haemagglutinin (HA), the unfavourable immunodominance of variable epitopes is a key challenge in immunization. We now know that HA has conserved, broadly neutralizing epitopes on its stem ${ }^{41-45}$. However, the immunodominance of variable, strainspecific epitopes on the HA head results in vaccine- and infection-elicited responses that are narrowly neutralizing, necessitating frequent changes in the strain composition of the antigens in influenza virus vaccines ${ }^{46}$. If we could decode the rules of antibody epitope immunodominance, we might learn to manipulate the antigen structure to render the conserved neutralizing epitopes immunodominant. Analyses of the human B cell repertoire and increasingly efficient physical and structural epitopemapping techniques could make investigation of the structural basis for epitope immunodominance, and the use of that information in vaccine design, much more tractable.

Recent investigations employing these techniques have yielded useful results. An antibody cloned from a human immune response to an influenza virus vaccine neutralized $\mathrm{H} 1$ influenza virus strains broadly, although there were 'drop-outs' of strains that escaped neutralization. X-ray crystallographic analysis of a complex between the antibody and the HA head revealed the basis for this pattern of broad neutralization ${ }^{47}$. In an example of molecular mimicry, complementaritydetermining loop 3 (CDR3) of the antibody reaches into the sialic acid-binding site of the HA head and binds the base of the pocket. The side chains of the amino acids in the antibody loop mimic the HA-interacting moieties of sialic acid with remarkable accuracy. Thus, the antibody does not bind the variable rim of the sialic acid-binding site, as do most HA head-specific antibodies, but rather docks on the conserved sialic acid-binding surface itself.

The discovery of a broadly neutralizing epitope on the HA stem has prompted widespread interest in engineering an HA that can elicit broadly neutralizing antibodies more efficiently ${ }^{48}$. This had been difficult because of the complexity of this epitope: it is formed by strands from both the HA1 and HA2 fragments of HA, which are brought 
into association through the overall folding of the multidomain pre-fusion conformer and which have different structural roles in the lower energy, post-fusion state. It has therefore proved challenging to convincingly recreate the stem structure as a singledomain construct for immunization studies. By contrast, the broadly neutralizing epitope at the base of the sialic acid-binding pocket is displayed on a single globular domain that is simple enough to be refolded from E. coli inclusion bodies and has a quantifiable function (haemagglutination) that can be assayed as an indicator of correct folding ${ }^{49-51}$. This tool might enable studies of how structure can be manipulated to focus antibody responses on the epitopes of greatest interest for vaccine design.

\section{Structural vaccinology realized}

In 2008, we wrote that the use of structural information in vaccine design would allow us to engineer more stable, homogeneous, efficiently produced vaccine antigens ${ }^{3}$. This ambition has guided vaccine research efforts in the intervening years, leading to the engineered RSV F subunit antigen, which is approaching clinical trials. Structural insights have also enabled the design of a GBS pilus-based fusion protein and an improved MenB single-domain fHbp antigen, both of which elicit immunity against more antigenic variants than their wild-type progenitors. The tools developed in these efforts will enable us to realize the longer-term vision of improving our understanding of the structural basis for immunogenicity and immunodominance in order to improve vaccine efficacy. Structural analysis has become a workhorse of practical vaccine design. We now routinely determine structures of vaccine antigens as a central task in vaccine optimization. The essential insights that antibody epitopes are conformational, extended protein surfaces and that short, floppy peptides rarely elicit protective antibodies are essential for selecting vaccine research strategies that have a high probability of success. Structure-based antigen design might ultimately enable us to succeed in overcoming some of the greatest remaining challenges in vaccinology, such as the development of an HIV $^{52}$ vaccine and a universal influenza virus vaccine $e^{45}$.

\section{Note added in proof}

A recent report from Tang and colleagues ${ }^{53}$ describes the structure-based design and evaluation of fHbps that are defective in factor $\mathrm{H}$ binding.
Philip R. Dormitzer is at Novartis Vaccines and Diagnostics Inc., 350 Massachusetts Avenue, Cambridge, Massachusetts 02139, USA.

Guido Grandi and Rino Rappuoli are at Novartis Vaccines and Diagnostics Srl, Via Fiorentina 1, 53100 Siena, Italy.

Correspondence to R.R. e-mail:rino.rappuoli@novartis.com

1. Rappuoli, R., Mandl, C. W., Black, S. \& De Gregorio, E. Vaccines for the twenty-first society. Nature Rev. Immunol. 11, 865-872 (2011).

2. Rappuoli, R. Reverse vaccinology. Curr. Opin. Microbiol. 3, 445-550 (2000)

3. Dormitzer, P. R., Ulmer, J. B. \& Rappuoli, R. Structure-based antigen design: a strategy for next generation vaccines. Trends Biotechnol. 26, 659-667 (2008).

4. Masignani, V. et al. Vaccination against Neisseria meningitidis using three variants of the lipoprotein GNA1870. J. Exp. Med. 197, 789-799 (2003).

5. Scarselli, M. et al. Rational design of a meningococcal antigen inducing broad protective immunity. Sci. Transl. Med. 3, 91 ra62 (2011).

6. Jiang, H. Q. et al. Broad vaccine coverage predicted for a bivalent recombinant factor $\mathrm{H}$ binding protein based vaccine to prevent serogroup $B$ meningococcal disease. Vaccine 28, 6086-6093 (2010)

7. Fletcher, L. D. et al. Vaccine potential of the Neisseria meningitidis 2086 lipoprotein. Infect. Immun. 72, 2088-2100 (2004).

8. Giuliani, M. M. et al. A universal vaccine for serogroup B meningococcus. Proc. Natl Acad. Sci. USA 103 10834-10839 (2006)

9. Mascioni, A. et al. Structural basis for the immunogenic properties of the meningococcal vaccine candidate LP2086. J. Biol. Chem. 284, 8738-8746 (2009).

10. Cantini, F. et al. Solution structure of the factor $\mathrm{H}$ binding protein, a survival factor and protective antigen of Neisseria meningitidis. J. Biol. Chem. 284, 9022-9026 (2009)

11. Schneider, M. C. et al. Neisseria meningitidis recruits factor $\mathrm{H}$ using protein mimicry of host carbohydrates. Nature 458, 890-893 (2009).

12. Giuntini, S., Reason, D. C. \& Granoff, D. M Complement-mediated bactericidal activity of antifactor $\mathrm{H}$ binding protein monoclonal antibodies against the meningococcus relies upon blocking factor $\mathrm{H}$ binding. Infect. Immun. 79, 3751-3759 (2011).

13. Scarselli, M. et al. Epitope mapping of a bactericidal monoclonal antibody against the factor $\mathrm{H}$ binding protein of Neisseria meningitidis. J. Mol. Biol. 386, 97-108 (2011).

14. Beernink, P. T. et al. A meningococcal factor $\mathrm{H}$ binding protein mutant that eliminates factor $\mathrm{H}$ binding enhances protective antibody responses to vaccination. J. Immunol. 186, 3606-3614 (2011).

15. Pajon, R., Beernink, P. T. \& Granoff, D. M. Design of meningococcal factor $\mathrm{H}$ binding protein mutant vaccines that do not bind human complement factor H. Infect. Immun. 80, 2667-2677 (2012)

16. Beernink, P. T. \& Granoff, D. M. Bactericidal antibody responses induced by meningococcal recombinant chimeric factor $\mathrm{H}$-binding protein vaccines. Infect. Immun. 76, 2568-2575 (2008).

17. Edmond, K. M., et al. Group B streptococcal disease in infants aged younger than 3 months: systematic review and meta-analysis. Lancet 379, 547-556 (2012).

18. Lin, F. C. et al. Level of maternal IgG anti-group B Streptococcus type III antibody correlated with protection of neonates against early-onset disease caused by this pathogen. J. Infect. Dis. 190, 928-934 (2004)

19. Lauer, P. et al. Genome analysis reveals pili in Group B Streptococcus. Science 309, 105 (2005).

20. Mora, M. et al. Group A Streptococcus produce piluslike structures containing protective antigens and Lancefield T antigens. Proc. Natl Acad. Sci. USA 102 15641-15646 (2005)

21. Telford, J. L., Barocchi, M. A., Margarit, I., Rappuoli, R. $\&$ Grandi, G. Pili in Gram-positive pathogens. Nature Rev. Microbiol.4, 509-519 (2006)

22. Margarit, l. et al. Preventing bacterial infections with pilus-based vaccines: the group B Streptococcus paradigm. J. Infect. Dis. 199, 108-115 (2009).
23. Nuccitelli, A. et al. Structure-based approach to rationally design a chimeric protein for an effective vaccine against Group B Streptococcus infections. Proc. Natl Acad. Sci. USA 108, 10278-10283 (2011)

24. Stockman, L. J., Corns, A. T., Anderson, L. J. \& Fischer-Langley, G. Respiratory syncytial virusassociated hospitalizations among infants and young children in the United States, 1997-2006. Pediatr. Infect. Dis. J. 31, 5-9 (2012).

25. Kim, H. W. et al. Respiratory syncytial virus disease in infants despite prior administration of antigenic inactivated vaccine. Am. J. Epidemiol. 89, 422-434 (1969).

26. Wright, P. F. et al. The absence of enhanced disease with wild type respiratory syncytial virus infection occurring after receipt of live, attenuated, respiratory syncytial virus vaccines. Vaccine $25,7372-7378$ (2007).

27. Swanson, K. A. et al. Structural basis for immunization with postfusion respiratory syncytial virus fusion $F$ glycoprotein (RSV F) to elicit high neutralizing antibody titers. Proc. Natl Acad. Sci. USA 108, 9619-9624 (2011).

28. McLellan, J. S., Yang, Y., Graham, B. S. \& Kwong, P. D. Structure of respiratory syncytial virus fusion glycoprotein in the postfusion conformation reveals preservation of neutralizing epitopes. J. Virol. 85, 7788-7796 (2011)

29. Yin, H. S., Wen, X., Paterson, R. G., Lamb, R. A. \& Jardetzky, T. S. Structure of the parainfluenza virus 5 $\mathrm{F}$ protein in its metastable, prefusion conformation. Nature 439, 38-44 (2006).

30. Magro, M. et al. Neutralizing antibodies against the preactive form of respiratory syncytial virus fusion protein offer unique possibilities for clinical intervention. Proc. Natl Acad. Sci. USA 109, 3089-3094 (2012).

31. Yin, H. S., Paterson, R. G., Wen, X., Lamb, R. A. \& Jardetzky, T. S. Structure of the uncleaved ectodomain of the paramyxovirus (hPIV3) fusion protein. Proc. Natl Acad. Sci. USA 102, 9288-9293 (2005).

32. Calder, L. J. et al. Electron microscopy of the human respiratory syncytial virus fusion protein and complexes that it forms with monoclonal antibodies. Virology 271, 122-131 (2000).

33. Langley, J. M. et al. A dose-ranging study of a subunit Respiratory Syncytial Virus subtype A vaccine with and without aluminum phosphate adjuvantation in adults $\geq 65$ years of age. Vaccine 27, 5913-5919 (2009).

34. Piedra, P. A. et al. Immunogenicity of a new purified fusion protein vaccine to respiratory syncytial virus: a multi-center trial in children with cystic fibrosis. Vaccine 21, 2448-2460 (2003).

35. McLellan, J. S. et al. Structural basis of respiratory syncytial virus neutralization by motavizumab. Nature Struct. Mol. Biol. 17, 248-250 (2010).

36. McLellan, J. S. et al. Structure of a major antigenic site on the respiratory syncytial virus fusion glycoprotein in complex with neutralizing antibody 101F. J. Virol. 84, 12236-12244 (2010).

37. López, J. A. et al. Conformational constraints of conserved neutralizing epitopes from a major antigenic area of human respiratory syncytial virus fusion glycoprotein. J. Gen. Virol. 74, 2567-2577 (1993).

38. Toiron, C. et al. Conformational studies of a short linear peptide corresponding to a major conserved neutralizing epitope of human respiratory syncytial virus fusion glycoprotein. Biopolymers 39, 537-548 (1996).

39. McLellan, J. S. et al. Design and characterization of epitope-scaffold immunogens that present the motavizumab epitope from respiratory syncytial virus. J. Mol. Biol. 409, 853-866 (2011).

40. Lingwood, D. et al. Structural and genetic basis for development of broadly neutralizing influenza antibodies. Nature 489, 566-571 (2012).

41. Okuno, Y., Isegawa, Y., Sasao, F. \& Ueda, S A common neutralizing epitope conserved between the hemagglutinins of influenza $\mathrm{A}$ virus $\mathrm{H} 1$ and $\mathrm{H} 2$ strains. J. Virol. 67, 2552-2558 (1993).

42. Ekiert, D. C. et al. Antibody recognition of a highly conserved influenza virus epitope. Science $\mathbf{3 2 4}$, 246-251 (2009)

43. Throsby, M. et al. Heterosubtypic neutralizing monoclonal antibodies cross-protective against $\mathrm{H} 5 \mathrm{~N} 1$ and $\mathrm{H} 1 \mathrm{~N} 1$ recovered from human $\mathrm{IgM}^{+}$memory $\mathrm{B}$ cells. PLOS ONE 3, e3942 (2008).

44. Sui, J. et al. Structural and functional bases for broad-spectrum neutralization of avian and human influenza A viruses. Nature Struct. Mol. Biol. 16, 265-273 (2009). 
45. Corti, D. et al. A neutralizing antibody selected from plasma cells that binds to group 1 and group 2 influenza A hemagglutinins. Science 333, 850-856 (2011).

46. Wiley, D. C., Wilson, I. A \& Skehel, J J Structural identification of the antibody-binding sites of Hong Kong influenza haemagglutinin and their involvemen in antigenic variation. Nature 289, 373-378 (1981).

47. Whittle, J. R. R. et al. Broadly neutralizing human antibody that recognizes the receptor-binding pocket of influenza virus hemagglutinin. Proc. Natl Acad. Sci. USA 108 14216-14221 (2011).

48. Steel, J. et al. Influenza virus vaccine based on the conserved hemagglutinin stalk domain. MBio 1 , e00018-e00010 (2010).

49. Liu, G. et al. Immunogenicity and efficacy of flagellin fused vaccine candidates targeting 2009 pandemic
H1N 1 influenza in mice. PLOS ONE 6, e20928 (2011).

50. Song, L. et al. Efficacious recombinant influenza vaccines produced by high yield bacterial expression a solution to global pandemic and seasonal needs. PLOS ONE 3, e2257 (2008).

51. Khurana, S. et al. Properly folded bacterially expressed $\mathrm{H} 1 \mathrm{~N} 1$ hemagglutinin globular head and ectodomain vaccines protect ferrets against $\mathrm{H} 1 \mathrm{~N} 1$ pandemic influenza virus. PLOS ONE 5, e 11548 (2011).

52. McLellan, J. S. et al. Structure of HIV-1 gp120 V1/V2 domain with broadly neutralizing antibody PG9. Nature 480, 336-343 (2011).

53. Johnson, S. et al. Design and evaluation of meningococcal vaccines through structure-based modification of host and pathogen molecules. $25 \mathrm{Oct}$ 2012 (doi:10.1371/journal.ppat.1002981)

\section{Acknowledgements}

The authors are grateful to M. Scarselli and F. Grassiccia for help with the figures, and to C. Mallia for help with the manuscript. The authors also acknowledge the partial contribution of the ADITEC Project (funded by the European Union Seventh Framework Programme, grant 280873) and of the grant P01AI089618 (funded by the National Institutes of Allergy and Infectious Diseases, US National Institutes of Health).

Competing interests statement

The authors declare competing financial interests; see Web version for details.

\section{FURTHER INFORMATION}

Protein Data Bank: http://www.rcsb.org/pdb/home/home.do PubMLST for fHBP: http://pubmlst.org/neisseria/fHbp

ALL LINKS ARE ACTIVE IN THE ONLINE PDF 